\title{
Ring-array photoacoustic tomography for imaging human finger vasculature
}

Misaki Nishiyama

Takeshi Namita

Kengo Kondo

Makoto Yamakawa

Tsuyoshi Shiina 


\title{
Ring-array photoacoustic tomography for imaging human finger vasculature
}

\author{
Misaki Nishiyama, ${ }^{*}$ Takeshi Namita, Kengo Kondo, Makoto Yamakawa, and Tsuyoshi Shiina \\ Kyoto University, Graduate School of Medicine, Department of Human Health Sciences, Kyoto, Japan
}

\begin{abstract}
For early diagnosis of rheumatoid arthritis (RA), it is important to visualize its potential marker, vascularization in the synovial membrane of the finger joints. Photoacoustic (PA) imaging, which can image blood vessels at high contrast and resolution, is expected to be a potential modality for earlier diagnosis of RA. In previous studies of PA finger imaging, different acoustic schemes, such as linear-shaped arrays, have been utilized, but these have limited detection views, rendering inaccurate reconstruction, and most of them require rotational detection. We are developing a PA system for finger vascular imaging using a ring-shaped array ultrasound (US) transducer. By designing the ring-array sensor based on simulations, using phantom experiments, it was demonstrated that we have created a system that can image small objects around 0.1 to $0.5 \mathrm{~mm}$ in diameter. The full width at half maximum of the slice direction of the system was within $2 \mathrm{~mm}$ and corresponded to that of the simulation. Moreover, we could clearly visualize healthy index finger vasculature and the location of the distal interphalangeal and proximal interphalangeal joints by PA and US echo images. In the future, this system could be used as a method for visualizing the three-dimensional vascularization of RA patients' fingers. () The Authors. Published by SPIE under a Creative Commons Attribution 4.0 Unported License. Distribution or reproduction of this work in whole or in part requires full attribution of the original publication, including its DOI. [DOI: 10.1117/1.JBO.24.9.096005]
\end{abstract}

Keywords: photoacoustic imaging; rheumatoid arthritis; ring-shaped ultrasound transducer; finger vascular imaging

Paper 190200R received Jun. 15, 2019; accepted for publication Aug. 20, 2019; published online Sep. 18, 2019.

\section{Introduction}

Rheumatoid arthritis (RA) is an inflammatory autoimmune disease that causes progressive articular and extra-articular destruction. It is estimated that $\sim 1 \%$ of the world population is affected by RA. A lack of appropriate diagnosis and treatment during the early stages leads to severe joint erosion and patients suffer from a reduced quality of life. ${ }^{1-3}$ Early effective treatment using medication, such as disease-modifying antirheumatic drugs, can alleviate the symptoms and reduce the risk of severe joint destruction. Although the 2010 American College of Rheumatology/European League Against Rheumatism criteria are now being applied as a means to diagnose RA, ${ }^{1,2}$ it remains quite difficult to identify the disease during the very early stage, which results in patients developing more severe symptoms. Therefore, the improvement of imaging techniques, which are a secondary part of the diagnosis, is needed to overcome these difficulties. ${ }^{4}$ Recently, for imaging RA diagnosis, it has been reported that inflammation of the synovial membrane in the joint, known as synovitis, is associated with vascularization that results in increasing the thickness of the synovial membrane and hypoxia, which can be effective RA markers. ${ }^{5-7}$ During its early stages, RA often appears in the finger joints. ${ }^{8}$ The imaging devices mainly used in clinical practice are conventional radiography (CR), magnetic resonance imaging (MRI), and Doppler ultrasound (US). ${ }^{9} \mathrm{CR}$ is sensitive to changes in joint spaces and bone erosion and is mainly applied during a later stage. MRI can visualize the synovitis, but it is expensive and requires contrast agents. Doppler US is widely used for assessing the vascularity of an inflamed synovium, but it is dependent on observational techniques and is difficult to quantify. ${ }^{4}$

*Address all correspondence to Misaki Nishiyama, E-mail: m.nishiyama@ shiina-lab.hs.med.kyoto-u.ac.jp
Photoacoustic (PA) imaging is expected to be a potential method that is complimentary to these imaging devices. This technique images light-absorbing structures in the tissue, such as blood vessels, by directing short nanosecond pulses at the skin surface. This leads to thermo-elastic expansion and propagation of US waves that are then detected using a US transducer. ${ }^{10-14}$ PA imaging can noninvasively image small vasculature in finger joints at high contrast without contrast agents using the wavelength-specific absorption of laser light by chromophores in the tissue; it could be an effective method for RA diagnosis. ${ }^{15}$ Moreover, by taking advantage of this optical imaging technique, multispectral PA imaging allows estimation of the oxygen saturation in blood vessels with the molar extinction coefficient of deoxyhemoglobin and oxyhemoglobin. This advantage can be applied to a quantitative evaluation of oxygenation in synovitis. ${ }^{16}$

In previous studies, PA imaging systems with a variety of acoustic detection schemes have been developed to accurately map finger joints. Using a PA system based on a linear array US transducer, $\mathrm{Xu}$ et al. ${ }^{17}$ measured healthy human peripheral joints and Jo et al. ${ }^{18}$ and van den Berg et al. ${ }^{19}$ succeeded in detecting joints affected by inflammatory arthritis. van Es et al. ${ }^{20-23}$ and Merčep et al. ${ }^{24}$ developed systems based on curvilinear geometry. Other systems had two or more single-element transducers rotating around the object. ${ }^{25,26}$ However, these approaches have limited detection views, resulting in inaccurate reconstructions, and most of them require rotational detection, which can take time and cause difficulty to patients. While Oeri et al. ${ }^{27}$ managed to design full-view tomography composed of four separate arc-like transducers, the image quality is relatively low owing to the different performance of each transducer and the geometry results in a more complex system. To address these difficulties, a ring-shaped US transducer has recently been developed, particularly for high-resolution small-animal 
whole-body imaging studies. ${ }^{28-32}$ This geometry can obtain all of the acoustic signals from around the object at once and improve its image quality by optimizing its potential to calculate the speed of sound distribution as a US-computed tomography. ${ }^{33}$ In addition, it can alternately and nearly simultaneously measure both PA and US signals, allowing it to realize accurate image synthesis without being affected by motion. Therefore, by applying it to finger imaging, an RA diagnosis that is more accurate can be realized, although until now studies have not been actively conducted.

Therefore, this study aimed to develop a PA system for smallfinger vascular imaging (i.e., 0.1 to $0.5 \mathrm{~mm}$ in diameter) using a ring-shaped US transducer. We designed the transducer based on simulations, investigated the imaging ability of the system in phantom experiments, and visualized a healthy human index finger vasculature.

\section{Design of the Ring-Array Sensor Based on Simulations}

\subsection{Numerical Models and Conditions}

Based on simulations, to realize a ring-array sensor able to clearly visualize the human finger vascular, we investigated the center frequency, element width and height of the sensor and the focal length of an acoustic lens for higher resolution of the slice direction. We set a simple finger vascular model, performed an ultrasonic-wave propagation simulation using $\mathrm{k}$-wave, ${ }^{34}$ obtained ultrasonic signals from the model, and then reconstructed them using the universal back projection (UBP) method $^{35}$ in MATLAB (2014a) in all simulations. First, we set up two numerical finger vascular models as shown in Fig. 1 to analyze the center frequency of the sensor. Figure 1(a) shows a cross-sectional vascular and bone model that indicates six different punctate blood vessels of 0.1 to $2 \mathrm{~mm}$ in diameter. The six vessels were $8.5 \mathrm{~mm}$ from the center of the ring array and at intervals of $30 \mathrm{deg}$. Figure 1(b) shows a branched blood vessel and bone model. The bone was at the center of the array as shown in Figs. 1(a) and 1(b). The number of elements was 256; the elemental pitch was $\sim 0.4 \mathrm{~mm}$; the ring diameter was $33 \mathrm{~mm}$, considering the finger diameter and acoustic lens; the number of grid points $(x-y$ axis) was $1750 \times 1750$; the lattice spacing ( $x-y$ axis) was $0.02 \mathrm{~mm}$; the attenuation coefficient

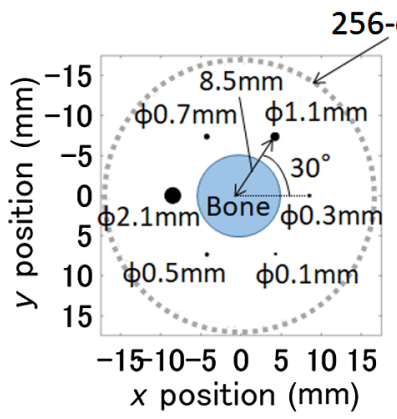

(a)

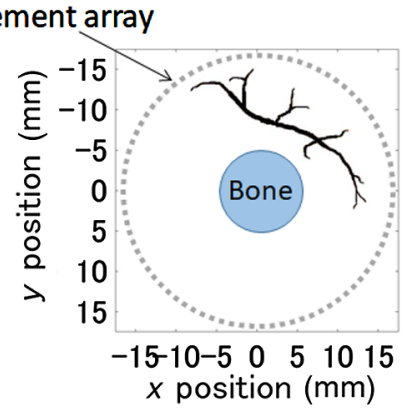

(b)
Fig. 1 The 2-D numerical finger vascular models. (a) Six different punctate blood vessels and bone model. Each vessel was $0.1,0.3$, $0.5,0.7,1.1$, and $2.1 \mathrm{~mm}$ in diameter, respectively, and $8.5 \mathrm{~mm}$ from the center of the ring array at intervals of $30 \mathrm{deg}$. (b) Branched blood vessel and bone model. In (a) and (b), a 256-element array was located around the model and the bone was $10 \mathrm{~mm}$ in diameter and at the center of the array. was $0.75 \mathrm{~dB} / \mathrm{MHz}^{1.5} \mathrm{~cm}$; the sound velocity was $1500 \mathrm{~m} / \mathrm{s}$; and the density was $1000 \mathrm{~kg} / \mathrm{m}^{3}$. The parameters of the bone shown in Fig. 1 were set such that the diameter was $10 \mathrm{~mm}$, the attenuation coefficient was $20 \mathrm{~dB} / \mathrm{MHz}^{1.5} \mathrm{~cm}$, the sound velocity was $4080 \mathrm{~m} / \mathrm{s}$, and the density was $1780 \mathrm{~kg} / \mathrm{m}^{3}$. We analyzed the center frequency by comparing the reconstructed images of both models at 2, 3, and $5 \mathrm{MHz}$. The fractional bandwidth was fixed at $80 \%$.

Next, we conducted a two-dimensional (2-D) simulation to investigate the element width of the circumferential direction of the ring-array sensor to clearly image the finger vasculature. In the simulation, the element width was assumed to be infinitesimal. Thus, we averaged several infinitesimal elements, regarded it as one element, and investigated the influence on the reconstructed image by comparing it with the image calculated using the infinitesimal element. As shown in Fig. 2, signals at 20 infinitesimal elements as shown by a green circle in Fig. 2(a) were integrated to approximately realize the signals at one element with a finite element width. In this case, the element width was $\sim 0.4 \mathrm{~mm}$. In the simulation, the model used was that shown in Fig. 2(b), which is the simple one without bone from Fig. 1(a), to make the influence of the element width on the image easier to understand. The other calculation parameters were the same as those of Fig. 1.

Third, we analyzed the element height of the ring-array sensor by evaluating the resolution of the slice direction using a three-dimensional (3-D) numerical model as shown in Fig. 3. For more accurate imaging, we considered the attachment of an acoustic lens to narrow the ultrasonic beam and investigated the appropriate focal length. Figure 3(a) shows the 3-D model. To assess the influence on the resolution in the case of several optical absorbers at different depths from the sensor, we set 11 spherical optical absorbers $0.5 \mathrm{~mm}$ in diameter in the homogeneous medium at 2.5 -mm intervals from an element on $y=$ 0 shown by the yellow thick line of the ring-array sensor. The element width on the $y=0$ of $z$ (slice) direction was defined as an element height. We conducted a simulation in a case in which the element height was 0 (infinitesimal) 2, 5, 7, 10, 15, and $20 \mathrm{~mm}$. The acoustic lens was attached inside the sensor. Figures 3(b) and 3(c) show the $x-y$ and $x-z$ cross-section views of Fig. 3(a), respectively. In Fig. 3(b), an element on $y=0$ is shown by a yellow circle. Figure 3(b) shows the positional relationship between optical absorbers and focal length. The focal lengths we considered in the analysis were $7.5,10,12.5$, and $15 \mathrm{~mm}$ from an element on $y=0$. In Fig. 3, the $z$-axis presents the slice direction. In the 3-D simulation, the number of grid points $(x-y-z$ axis) was $350 \times 350 \times 128$, the lattice spacing $(x-y-z$ axis) was $0.1 \mathrm{~mm}$, the ring diameter was $30 \mathrm{~mm}$ for easier analyses, and the other parameters were the same as those in Fig. 1.

To evaluate the resolution, an $x-z$ cross-sectional PA reconstruction image was calculated and then the full width at half maximum (FWHM) was calculated from the profile computed by plotting the maximum value of the PA amplitude from each absorber within the gray-dotted square in the $x-z$ crosssectional image shown in Fig. 3(d). At each element height and focal length, the FWHM was computed and the value plotted on a 2-D map.

\subsection{Results}

Figure 4 shows reconstructed images of Fig. 1 at each center frequency (fractional bandwidth $80 \%$ ). Figures $4(a)-4(c)$ are the 


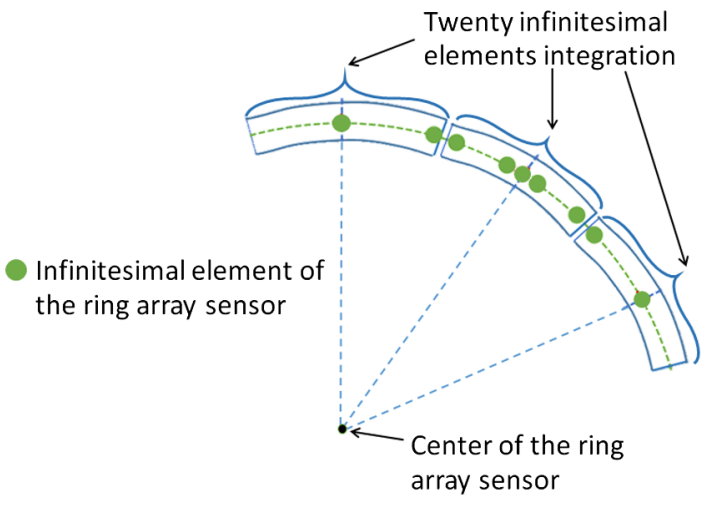

(a)

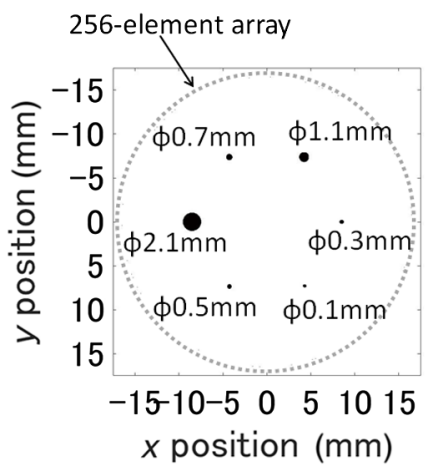

(b)

Fig. 2 Investigation of the design of the element width of the ring-array sensor. (a) Element width used in the analysis. Twenty infinitesimal elements were integrated and regarded as one element. In this case, the element width was $\sim 0.4 \mathrm{~mm}$. (b) Finger vascular model, which is the simple model without bone from Fig. 1(a).

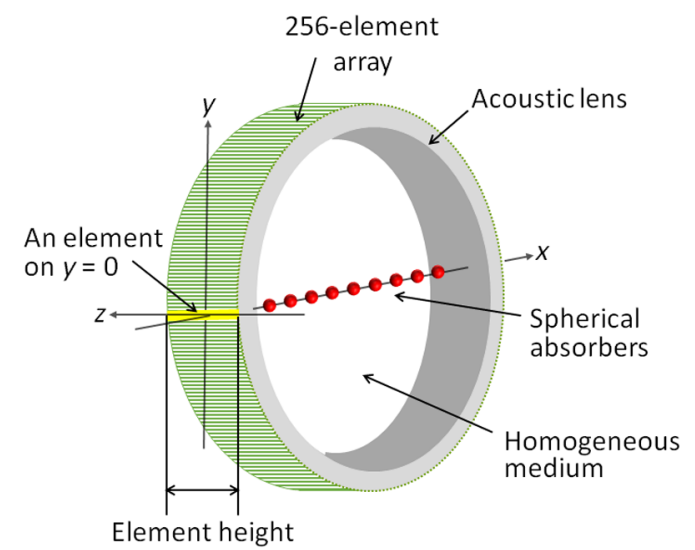

(a)

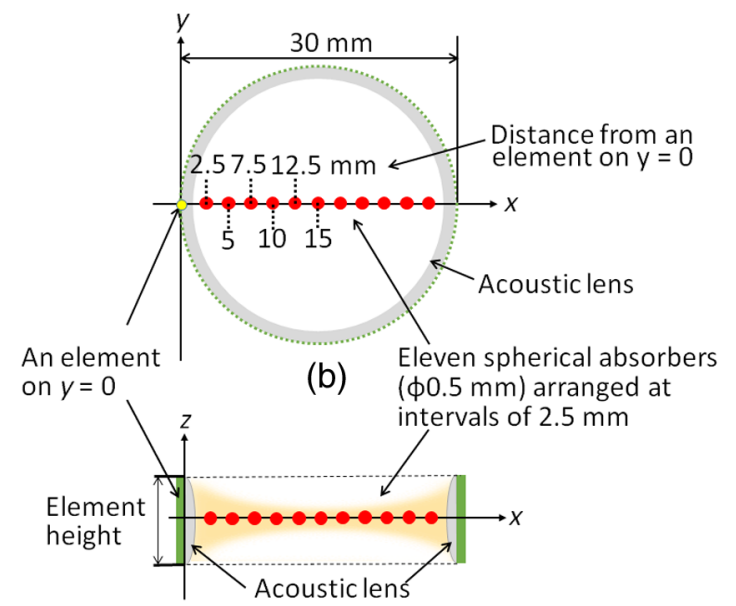

(c)

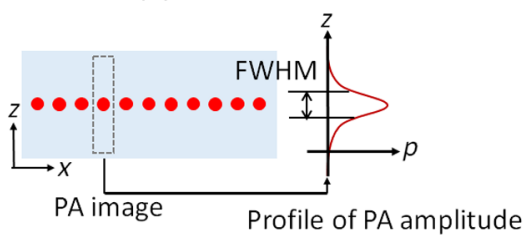

(d)

Fig. 3 Analysis of the design of the element height of the ring-array sensor and the focal length of the acoustic lens using a 3-D numerical model. $Z$-axis shows the slice direction. (a) A 3-D numerical model. Eleven 0.5 -mm-diameter spherical absorbers were arranged in the homogeneous medium at $2.5-\mathrm{mm}$ intervals from an element on $y=0$ shown by the yellow thick line of the ring-array sensor. The element width on $y=0$ in the $z$ direction was defined as an element height. The acoustic lens was attached inside the sensor. (b) $x-y$ cross-sectional view of (a). A yellow circle showing the element on $y=0$. (c) $x-z$ cross-sectional view of (a) showing the positional relationship between the optical absorbers and focal length. The focal lengths we considered in the analysis were $7.5,10,12.5$, and $15 \mathrm{~mm}$ from an element on $y=0$. (d) Method for evaluating the slice direction resolution. The $x-z$ cross-sectional PA image was reconstructed and then FWHM was calculated from the profile computed by plotting the maximum value of the PA amplitude from each absorber within the gray-dotted square in the cross-sectional image ( $p$ : amplitude).

result of Fig. 1(a), and Figs. 4(d)-4(f) are the result of Fig. 1(b). In both models, the signals from the blood vessels at 2 and $3 \mathrm{MHz}$ were stronger than those at $5 \mathrm{MHz}$. In the images at $2 \mathrm{MHz}$, ringing and artifacts from acoustic reflection of the bone were notably observed, while at $3 \mathrm{MHz}$, ringing and artifacts were controlled more than at $2 \mathrm{MHz}$. In addition, the thin parts of the branched vessel shown by a red-dotted circle were more clearly visualized at $3 \mathrm{MHz}$ than at $2 \mathrm{MHz}$. To investigate the image of each frequency in more detail, a PA amplitude of each blood vessel shown in Figs. 4(a)-4(c) was calculated and plotted by the center frequency as shown in Fig. 5. For example, in the reconstructed image at $3 \mathrm{MHz}$ shown in Fig. 5(a), the same as 


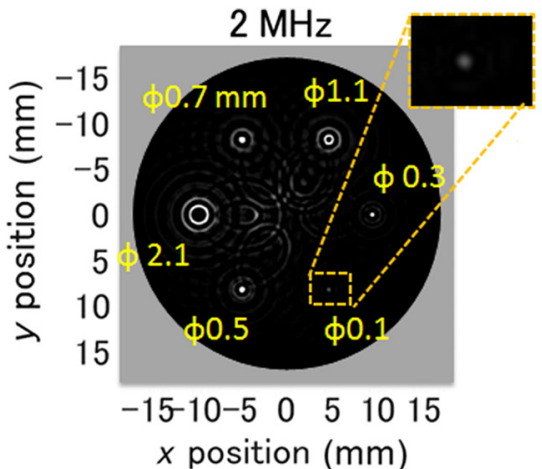

(a)

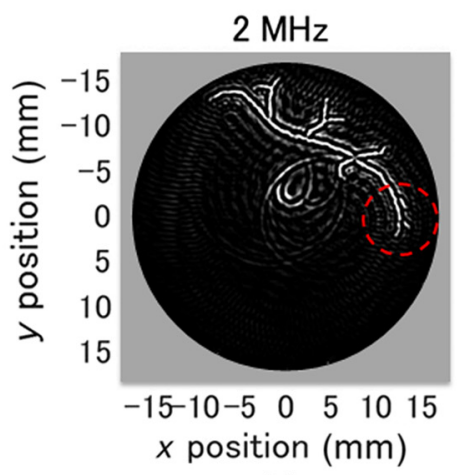

(d)

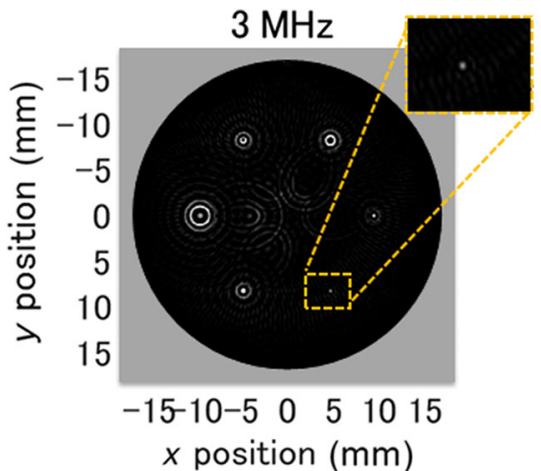

(b)

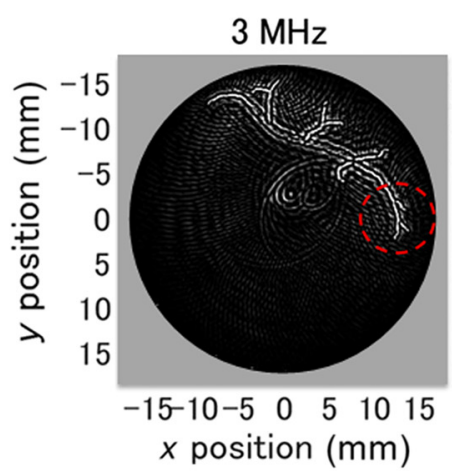

(e)

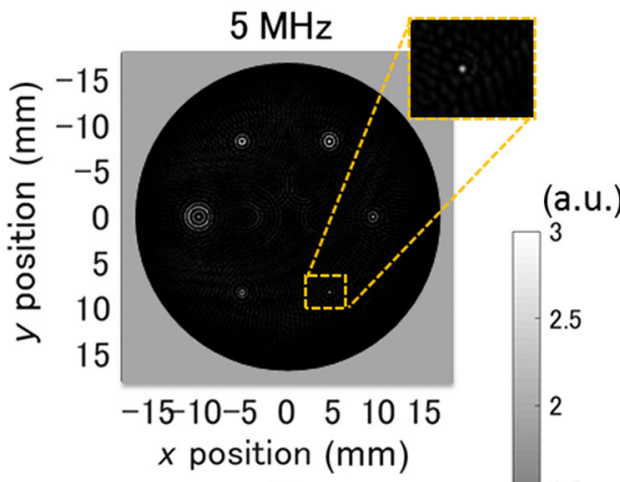

(c)

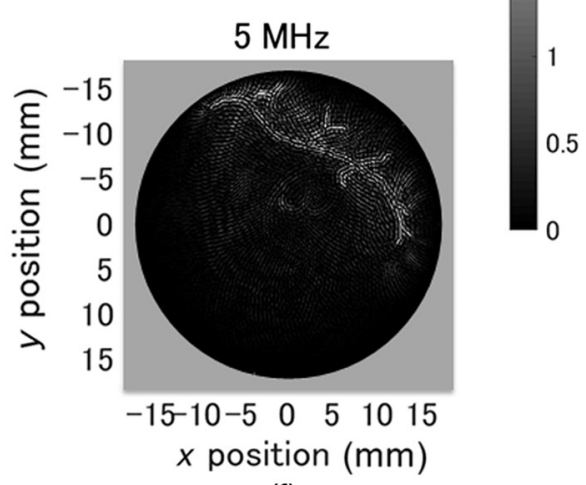

(f)

Fig. 4 Reconstructed images for each center frequency of the two models. (a)-(c) The results of Fig. 1(a), and (d)-(f) the results of Fig. 1(b). Each orange-dotted square in (a)-(c) shows magnification of the image of the 0.1 -mm-diameter blood vessel. The red-dotted circle in (d) and (e) shows the thin parts of the blood vessel.

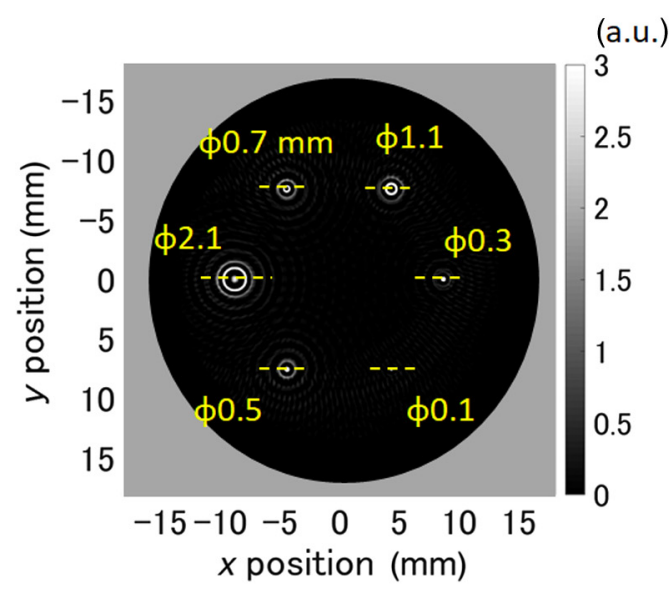

(a)

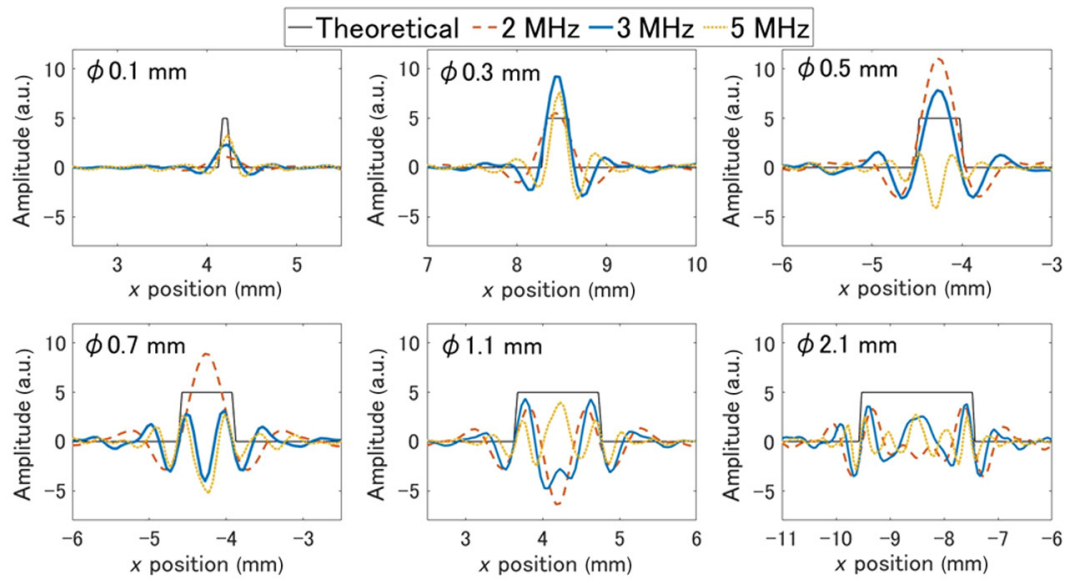

(b)

Fig. 5 (a) Position where the PA amplitude of each blood vessel was calculated shown by the yellowdotted line using the reconstructed image at $3 \mathrm{MHz}$, the same as Fig. 4(b). (b) PA amplitude of each blood vessel along the yellow-dotted line parallel to the $x$-axis in (a).

Fig. 4(b), the PA amplitude of each blood vessel along the yellow-dotted line parallel to the $x$-axis was plotted. The amplitude in the other frequencies was also plotted in the same manner. The results are shown in Fig. 5(b). The black, brown, blue, and yellow lines show the theoretical, 2-, 3-, and 5-MHz distributions, respectively. From Fig. 5(b), at $2 \mathrm{MHz}$, one cannot clearly visualize a $0.1-\mathrm{mm}$-diameter blood vessel, while at 3 and
$5 \mathrm{MHz}$, one can image a 0.1-mm-diameter blood vessel. However, $5 \mathrm{MHz}$ has difficulty clearly imaging a greater than 0.5-mm-diameter blood vessel as shown in Figs. 4(c) and 5(b). In this study, we aimed to design a ring-array sensor that is able to visualize relatively larger blood vessels $(\sim 1$ to $2 \mathrm{~mm}$ in diameter) as well as smaller vessels $(\sim 0.1 \mathrm{~mm})$ because the size of the RA neovascularity is unclear. Therefore, $3 \mathrm{MHz}$, at which 


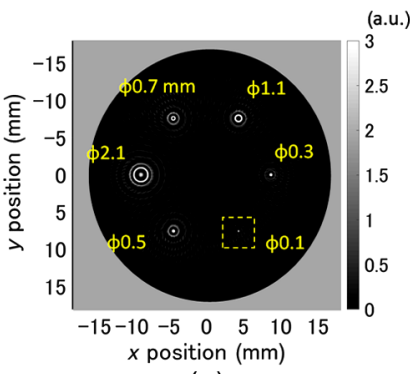

(a)

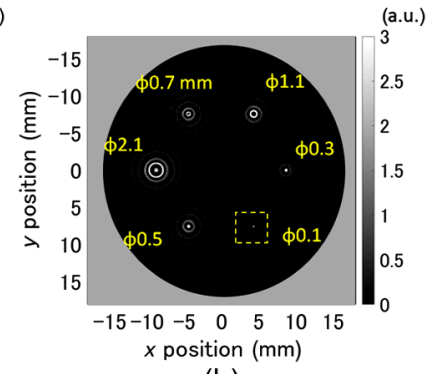

(b)
Fig. 6 Reconstructed images in each element width. The center frequency and fractional bandwidth used in the calculation were $3 \mathrm{MHz}$ and $80 \%$, respectively. (a) A result calculated by infinitesimal elements. (b) A result calculated by averaging 20 infinitesimal elements. Each 0.1 -mm-diameter blood vessel is marked by a yellow-dotted square as shown in Fig. 7.

one can visualize 0.1 to $2.1 \mathrm{~mm}$ relatively clearly and control the ringing and artifacts, was selected as the appropriate center frequency of the ring-array sensor.

Figure 6 shows the reconstructed images at each element width. The center frequency and fractional bandwidth used in the calculation were $3 \mathrm{MHz}$ and $80 \%$, respectively. Figure 6(a) shows the result by infinitesimal elements, and Fig. 6(b) shows the result of averaging 20 infinitesimal elements. In Figs. 6(a) and $6(\mathrm{~b})$, the difference cannot be seen. Figure 6(b) shows the element width calculated by averaging 20 infinitesimal elements, which does not affect the image.

In addition, the amplitude distribution of a 0.1-mm-diameter blood vessel shown in Fig. 6(a) and 6(b) was calculated as shown in Fig. 7. Figures 7(a) and 7(b) presents the magnification of the $0.1-\mathrm{mm}$-diameter blood vessel shown by the yellowdotted square in Figs. 6(a) and 6(b), respectively. Similar to Fig. 5, each amplitude along the yellow-dotted line in Figs. 7(a) and 7(b) parallel to the $x$-axis was plotted. The result is shown in Fig. 7(c). Although a difference in the maximum of each amplitude was observed, the FWHM of the profile in infinitesimal elements and 20 infinitesimal elements integration was 0.23 and $0.24 \mathrm{~mm}$, respectively. The error was negligibly small. Therefore, we predicted the element width calculated in the case of a close arrangement of each element will not affect the reconstructed image.
Figure 8(a) shows the FWHM distribution corresponding to each focal length, element height, and distance from an element on $y=0$. From Fig. 8(a), the element height of $5 \mathrm{~mm}$ stabilized a relatively low FWHM regardless of the distance from the element (depth) and maintained it within a few millimeters with focal lengths of 12.5 and $15 \mathrm{~mm}$. We calculated the FWHM and peak value of the pressure amplitude corresponding to each focal length and the distance from an element on $y=0$, fixing the element height to $5 \mathrm{~mm}$. The results are shown in Figs. 8(b) and $8(\mathrm{c})$. At $12.5 \mathrm{~mm}$, the shallower part tended to have a narrower half width and larger amplitude, while at $15 \mathrm{~mm}$, the deeper part did. We presumed that $12.5 \mathrm{~mm}$ would be better for finger imaging. We predicted those values cannot change when the ring diameter is $33 \mathrm{~mm}$. We therefore determined an element height $(5 \mathrm{~mm})$ and focal length $(12.5 \mathrm{~mm})$ that could maintain the FWHM in the slice direction within $\sim 2 \mathrm{~mm}$; that is, these values can realize a higher resolution in the slice direction.

\section{Phantom Experiments}

\subsection{Imaging Setup}

Figure 9 shows the imaging setup used during the experiment. We created a ring-shaped optical illumination imaging system because it could homogenously and strongly illuminate the object. $^{28-33}$ A photograph of the sensor and optical illuminator appears in the blue-dotted square on the upper left side of Fig. 9.

Each designed value of the ring-array sensor provided by the simulation result was the following. The ring diameter was $33 \mathrm{~mm}$; the number of elements was 256; and the element pitch, width, and height were $0.4,0.3$, and $5 \mathrm{~mm}$, respectively. The measured value of the center frequency and fractional bandwidth were $2.8 \mathrm{MHz}$ and $85 \%$, respectively. The approximate ring-shaped illumination is indicated by a red-dotted square on the lower left side. The ring-shaped light was illuminated onto the phantom model through an optical fiber. Optical illumination by 6-ns laser pulses was generated by a combination of an Nd:YAG laser (EKSPLA, NL313-30-SH, $532 \mathrm{~nm}, 30 \mathrm{~Hz}$ ) pumping an optical parametric oscillator (EKSPLA, PG-152B$\mathrm{T}, 50-150 \mathrm{~mJ})$. The system was set up such that the signal was output from the laser to the trigger and obtained at a sampling frequency of $11.4 \mathrm{MHz}$ by the data acquisition system (Verasonics). The measurement was performed at $800 \mathrm{~nm}$.

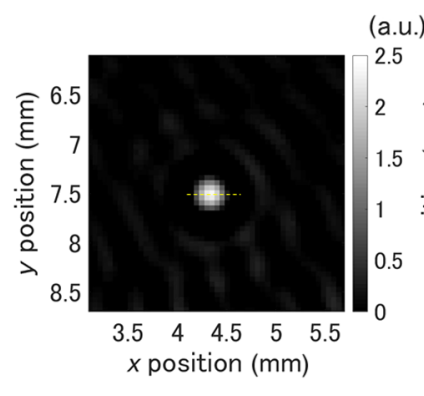

(a)

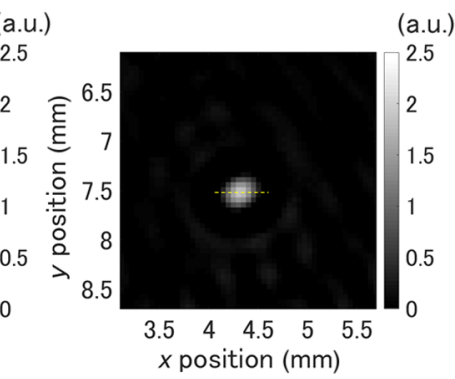

(b)

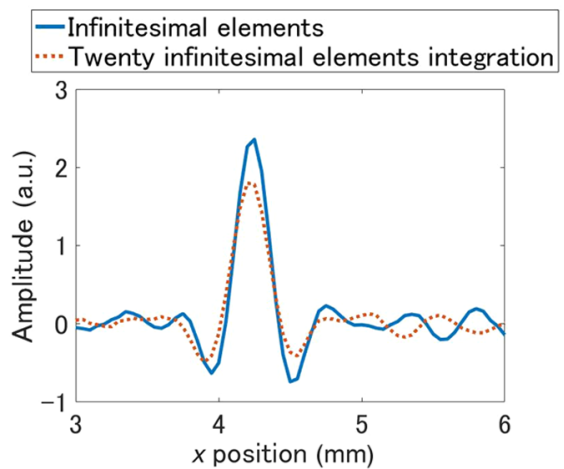

(c)

Fig. 7 (a) Magnification of the 0.1-mm-diameter blood vessel shown by the yellow-dotted square in Fig. 6(a). (b) Magnification of the $0.1-\mathrm{mm}$-diameter blood vessel shown by the yellow-dotted square in Fig. 6(b). (c) Amplitude distribution of the 0.1-mm-diameter blood vessel along the yellow-dotted line in (a) and (b) parallel to the $x$-axis. The blue line shows the case of infinitesimal elements and the orange line the case of 20 infinitesimal elements integrated. 

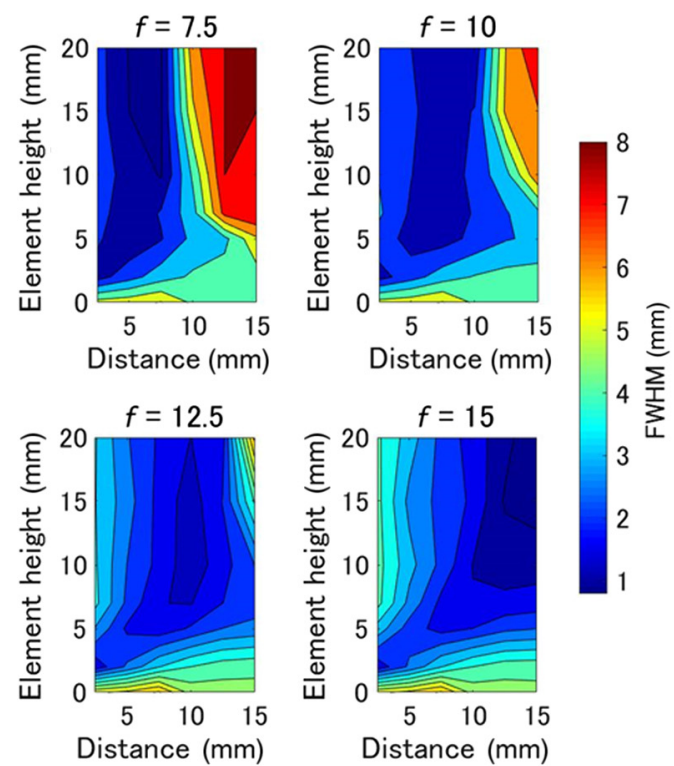

(a)
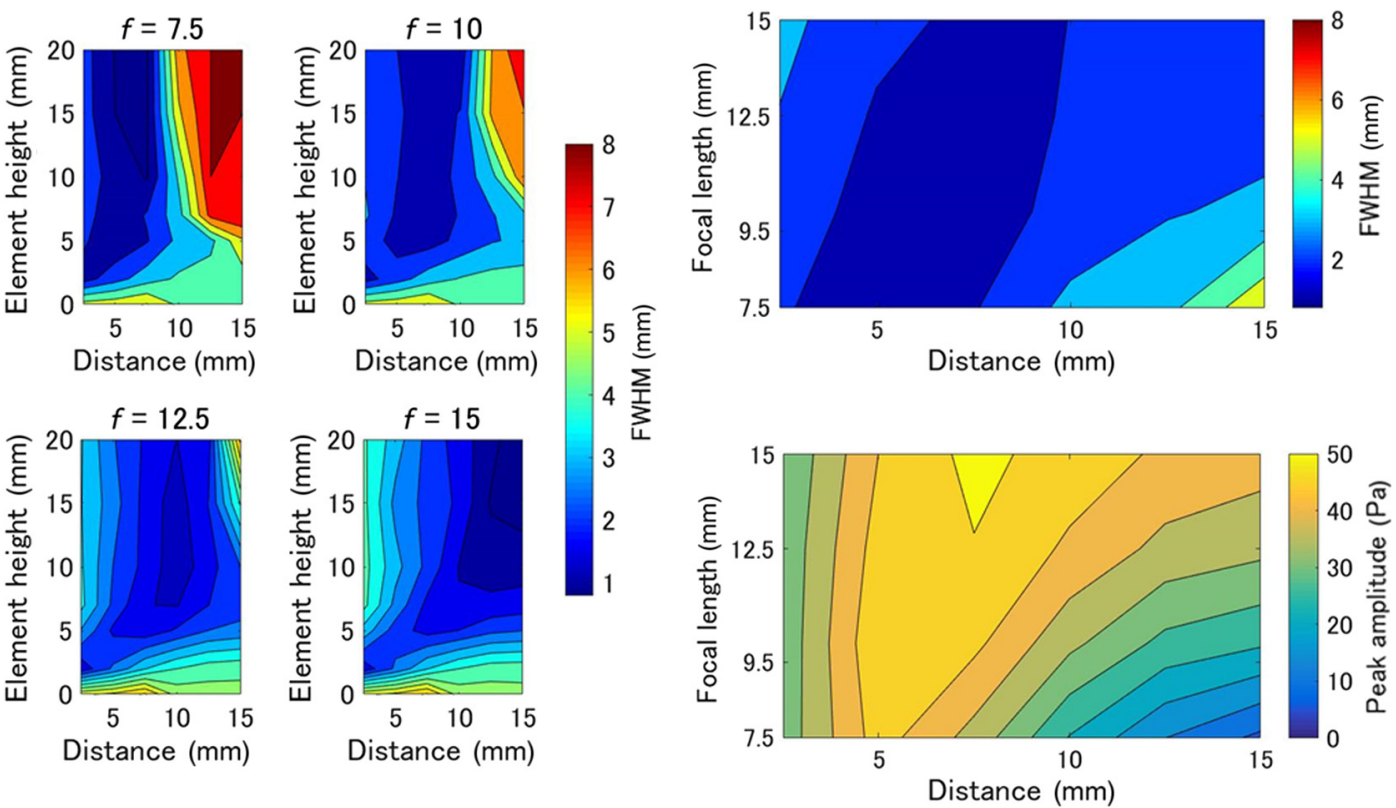

(b)

Fig. 8 FWHM distribution corresponding to each focal length, element height, and distance from an element on $y=0$. The element height of $5 \mathrm{~mm}$ stabilized a relatively low FWHM regardless of the distance from the element. (b) FWHM and (c) peak value of the pressure amplitude corresponding to each focal length and distance with the element height fixed to $5 \mathrm{~mm}$. ( $f$ : focal length).

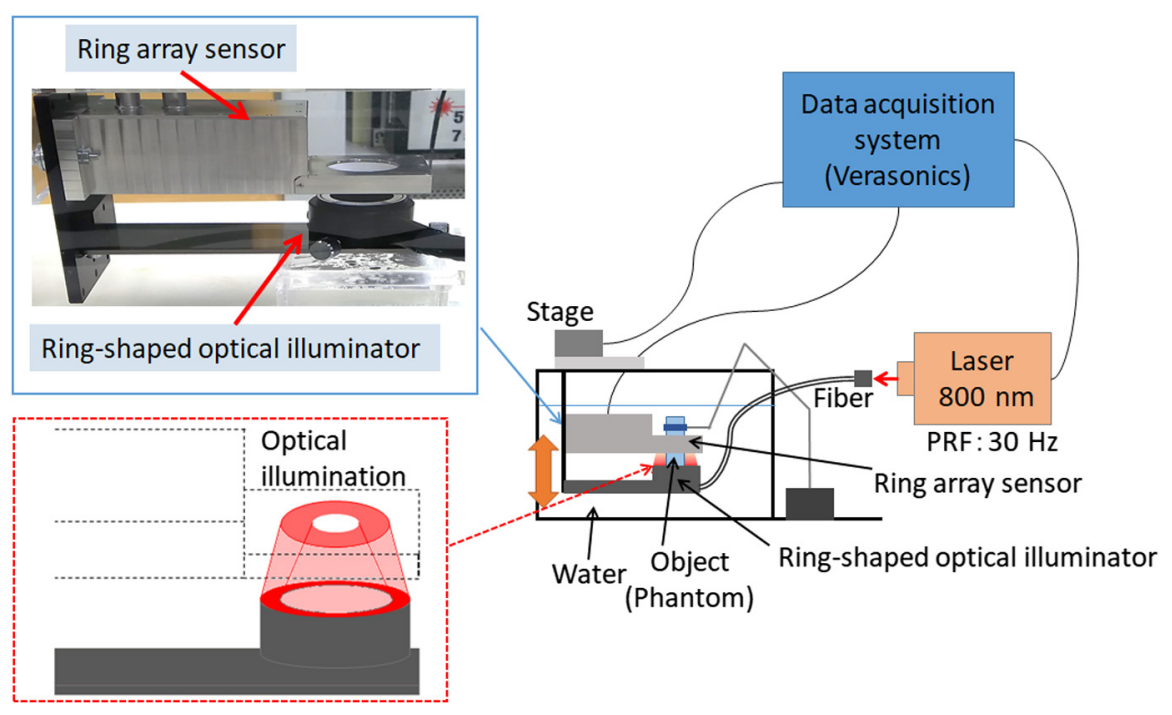

Fig. 9 Diagram of the imaging system. The upper left side (blue-solid square) is a photograph of the ringarray sensor and ring-shaped optical illuminator; the lower left side (red-dotted square) illustrates the estimated illumination shape. The orange arrow shows the mobile direction of the sensor and illuminator. The object was set in the center of the ring-array sensor and the optical illuminator in a water tank. The ring-shaped light $(800 \mathrm{~nm})$ was illuminated onto it through an optical fiber. PA and US signals were simultaneously received by the sensor and processed by a data acquisition system (Verasonics).

The averaged light intensity at the surface of the illuminator was $\sim 16.5 \mathrm{~mJ}$ and the estimated optical fluence was $\sim 2.3 \mathrm{~mJ} / \mathrm{cm}^{2}$, which was within the safety limit. The system can simultaneously receive both PA and US signals. Using this system, a 2-D imaging experiment was conducted by placing the phantom in the center of the sensor in a water tank. Additionally, a 3-D measurement to calculate the FWHM of the slice direction, important for accurate 3-D imaging, was conducted by simultaneously moving both the sensor and illuminator perpendicular to the cross section as indicated by the orange arrow in Fig. 9.

\subsection{Phantom Models}

To verify the sensor ability to clearly visualize multiple absorbers that had different diameters of $\sim 0.1$ to $1 \mathrm{~mm}$, simple 


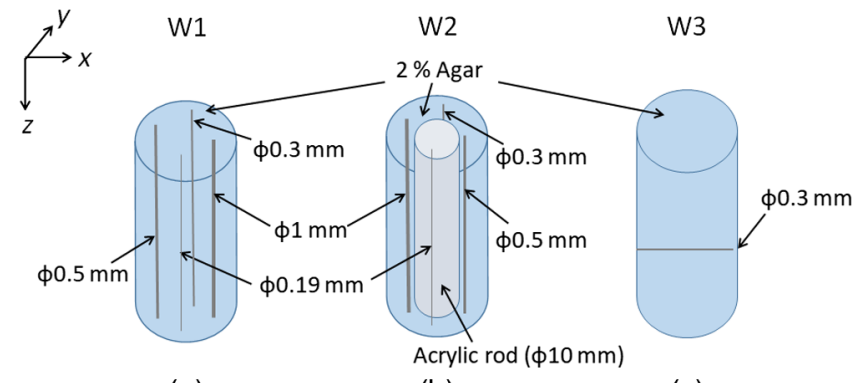

(a)

(b)

(c)

Fig. 10 Cylindrical $2 \%$ agar phantom models to verify sensor ability to clearly image absorbers and the slice direction resolution (a) W1 phantom was embedded with wires that had diameters of 0.19 , $0.3,0.5$, and $1 \mathrm{~mm}$ mimicking blood vessels. (b) W2 phantom was embedded with wires of the same size as W1 and a 10-mm-diameter transparent acrylic rod mimicking a bone. (c) W3 phantom was embedded with a 0.3-mm-diameter wire to evaluate the slice direction resolution.

cylindrical phantom models mimicking a finger were fabricated as shown in Figs. 10(a) and 10(b). In addition, we created a simple phantom model as shown in Fig. 10(c) to evaluate the slice direction resolution by 3-D scanning and compared it with the simulation results (Fig. 8).

Phantom (W1), shown in Fig. 10(a), was composed of $2 \%$ agar gel embedded wires with diameters of $0.19,0.3,0.5$, and $1 \mathrm{~mm}$ mimicking blood vessels. To better mimic a finger, phantom (W2), shown in Fig. 10(b), was composed of embedded wires of the same diameters as W1 and a transparent acrylic rod with a diameter of $10 \mathrm{~mm}$ mimicking the bone. In addition, a phantom model (W3) to evaluate the slice direction resolution was embedded with a $0.3-\mathrm{mm}$-diameter wire parallel to its minor axis as shown in Fig. 10(c).

\subsection{Cross-Sectional Images}

Figure 11 presents a cross-sectional PA image of W1 and an amplitude profile of each wire compared with the simulation.
The PA image was reconstructed using the UBP method. The profile was calculated by plotting the maximum value of the PA amplitude on the $y$-axis within each blue-dotted square shown in Fig. 11(a). Figure 11(a) shows that the system can image multiple optical absorbers of different diameters including $0.19 \mathrm{~mm}$. Figure 11(b) shows that the distribution during the experiment was approximately the same as that during the simulation. In addition, the FWHM was computed for a $0.19-\mathrm{mm}-$ diameter wire. The experimental value was $0.275 \mathrm{~mm}$, nearly the same as the value of $0.245 \mathrm{~mm}$ calculated by the simulation.

The designed array sensor can simultaneously receive both PA and US signals; thus, a coregistered PA and US echo image of the W2 phantom can be acquired. The US echo image was reconstructed using a synthetic-aperture US imaging method. ${ }^{36}$ Figure 12(a) shows a PA image of the W2 phantom, which can also accurately visualize optical absorbers, including those that are $0.19 \mathrm{~mm}$ in diameter as shown in Fig. 11(a). Figure 12(b) shows the pseudocolor PA image superimposed on a grayscale US echo image, in which the contrast and brightness were manually enhanced. In Fig. 12(b), the relationships between the four wires and the acrylic rod are clearly shown, indicating that the system has the potential to provide useful information around joints.

\subsection{Three-dimensional Scanning Results}

The W3 phantom shown in Fig. 10(c) was placed in the center of the system shown in Fig. 9 and was imaged by scanning both the array sensor and optical illuminator at $0.1-\mathrm{mm}$ intervals up to $15 \mathrm{~mm}$ perpendicular to the cross section. The imaging time per slice with 10 signal averages was $\sim 1.8 \mathrm{~s}$. To verify the potential to narrow the beam width in the slice direction, we conducted experiments with and without an acoustic lens (focal length of $12.5 \mathrm{~mm}$ ). We calculated the FWHM for the PA amplitude of the wire in the slice direction. Figure 13 shows the calculation method with the acoustic lens. Similar to the simulation, we reconstructed $y-z$ cross-sectional images [Fig. 13(b)] of the wire at several points on the $x$-axis in its $x-y$ image

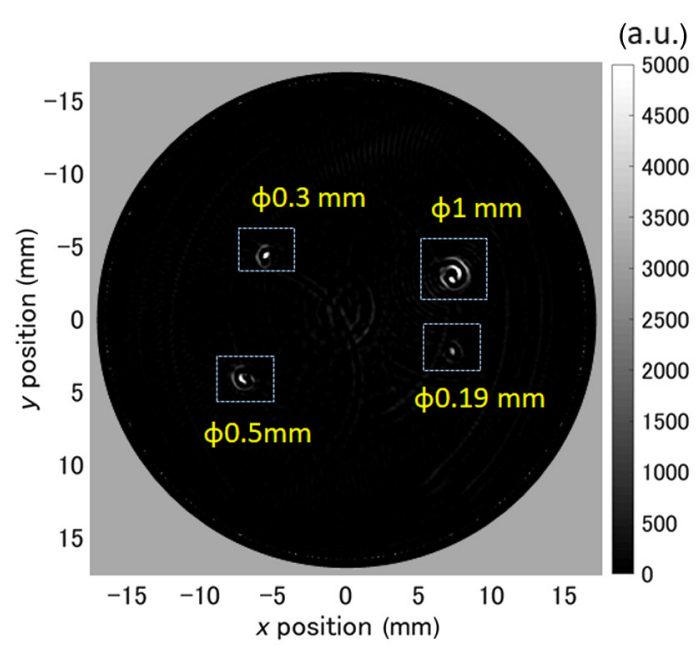

(a)
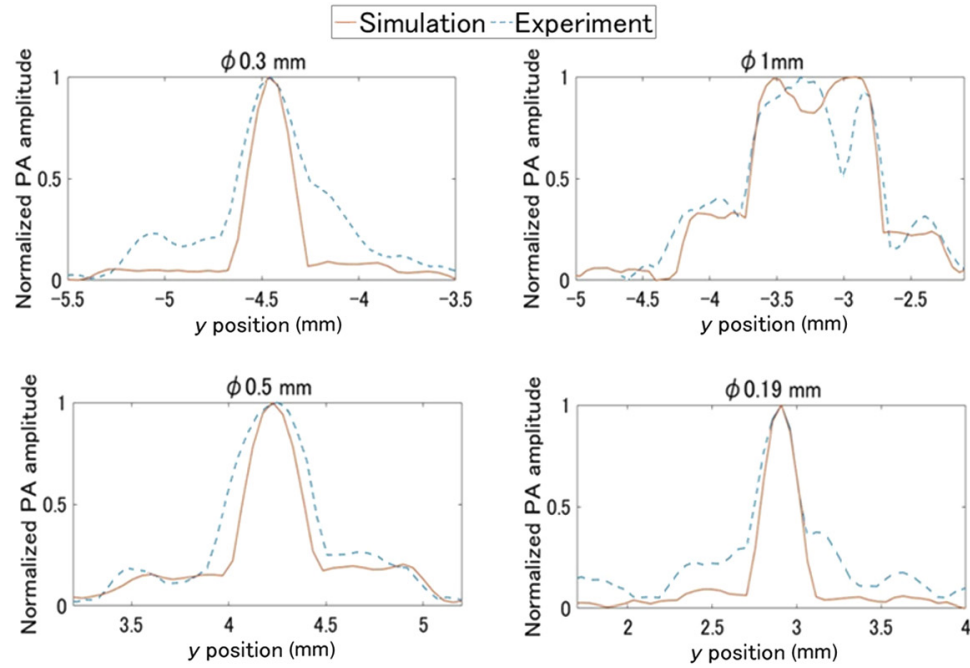

(b)

Fig. 11 Cross-sectional PA image and amplitude profile of W1 phantom. (a) PA image. (b) PA amplitude distribution of each wire compared with that of the simulation. The experimental profile was calculated by plotting the maximum value of the PA amplitude on the $y$-axis within each blue-dotted square in the PA image of W1. 


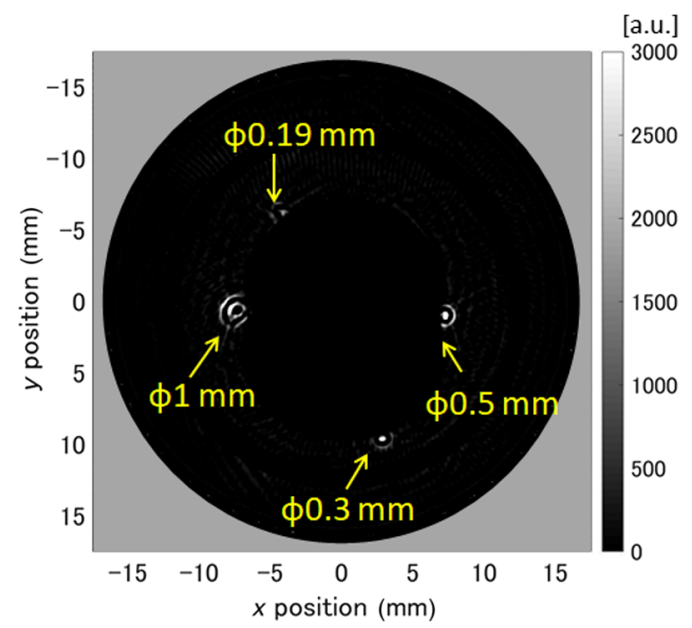

(a)

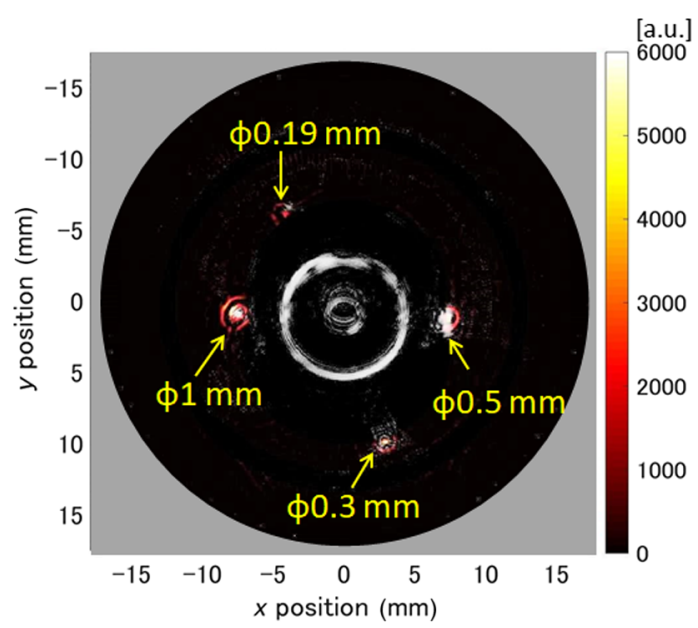

(b)

Fig. 12 Cross-sectional image of W2 phantom. (a) PA image. (b) Pseudocolor PA image superimposed on a US echo image. The contrast and brightness were manually enhanced. Each yellow arrow shows the wire diameter.

(a.u.)

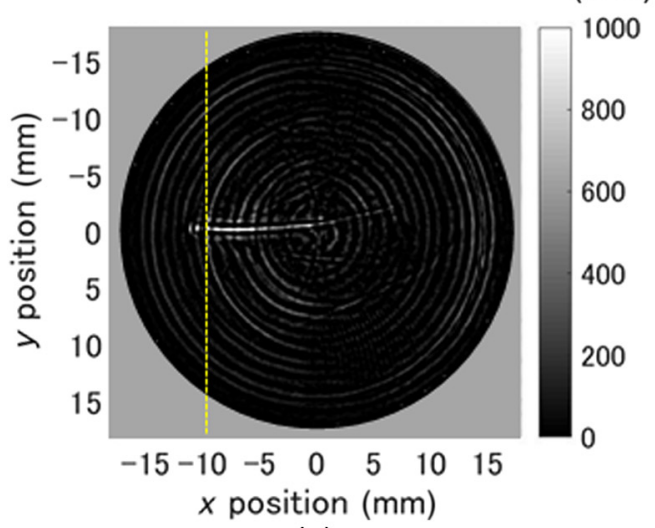

(a)

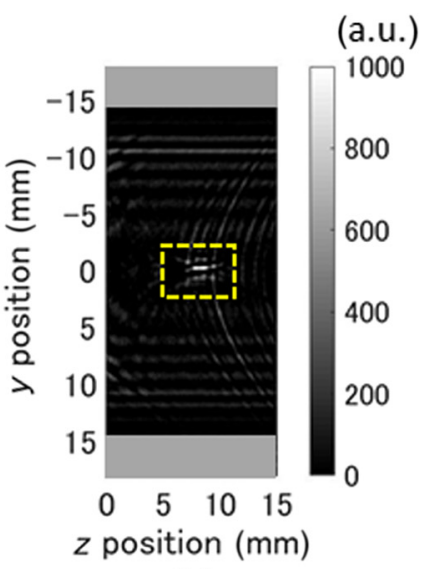

(b)

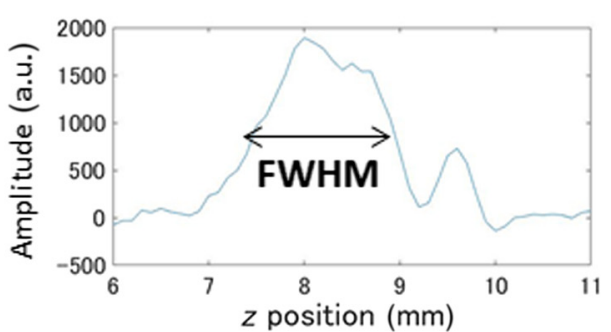

(c)

Fig. 13 Method of calculating the FWHM for the wire PA amplitude. (a) $x-y$ cross-sectional image. (b) $y-z$ cross-sectional image at the yellow-dotted line in the $x-y$ image. (c) PA amplitude profile of the wire within the yellow-dotted square in the $y-z$ image.

[Fig. 13(a)] and computed the FWHM for its PA amplitude profile [Fig. 13(c)] at each position. We then plotted the values from the experiment and simulation corresponding to the distance from the element on $y=0$ and evaluated the FWHM. Figure 14 shows that the FWHM for the experiment tended to vary as did that of the simulation. It also shows that a system

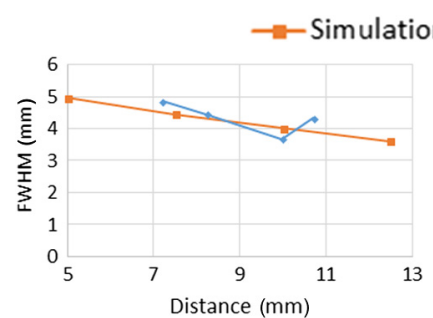

(a)

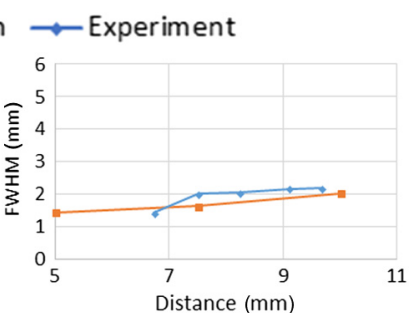

(b)
Fig. 14 Variations in FWHM corresponding to the distance from the element on $y=0$. (a) Without acoustic lens and (b) with acoustic lens (focal length $=12.5 \mathrm{~mm}$ ). with an acoustic lens with a focal length of $12.5 \mathrm{~mm}$ can maintain the FWHM to around half as narrow as a system without an acoustic lens, providing higher slice direction resolution, similar to the simulation.

\section{Finger Imaging}

\subsection{Imaging Setup}

To confirm the ability of the system to visualize small biological vascular structures, we acquired a signal from a healthy human index finger (author K. Kondo) at $800 \mathrm{~nm}$ using the same system shown in Fig. 9. The index finger was set in the center of the ring-array sensor and optical illuminator (Fig. 15). The experiment was conducted by scanning both the ring-array sensor and the illuminator at $0.1-\mathrm{mm}$ intervals up to $30 \mathrm{~mm}$ perpendicular to the cross section, as performed during the 3-D phantom experiments.

The data acquisition time per slice was $\sim 0.033 \mathrm{~s}$ and the total acquisition time it takes to complete the $30-\mathrm{mm}$ measurement 


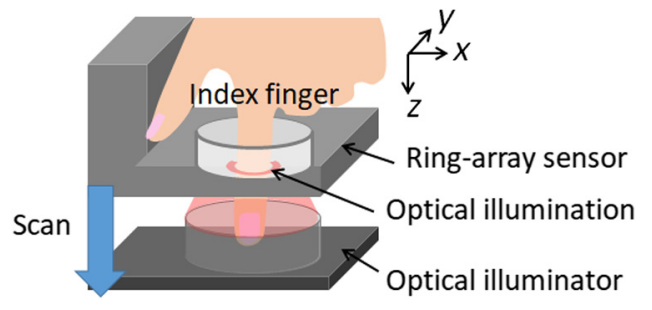

Fig. 15 Diagram of index finger imaging. The index finger was set in the center of the ring-array sensor and optical illuminator and scanned at $0.1-\mathrm{mm}$ intervals up to $30 \mathrm{~mm}$ using the same system as the phantom experiments.

was $\sim 10 \mathrm{~s}$. The first scan was near the distal interphalangeal (DIP) joint and the second was near the proximal interphalangeal (PIP) joint. We simultaneously received PA and US signals from the system with an acoustic lens with a focal length of $12.5 \mathrm{~mm}$ because it can provide a higher resolution in the axial direction.

\subsection{Vascular Images}

Figures 16 and 17 show the results of the measurements around the DIP and PIP joints, respectively. Figures 16(a) and 16(d) [Figs. 17(a) and 17(d)] show cross-sectional images, and Figs. 16(b), 16(c), 16(e), and 16(f) [Figs. 17(b), 17(c), 17(e), and 17(f)] show maximum intensity projection (MIP) images around the DIP (PIP) joint of the index finger. MIP images were calculated from the skin surface to the center of the finger. Figures 16(a)-16(c) [Figs. 17(a)-17(c)] show PA images, and Figs. 16(d)-16(f) [Figs. 17(d)-17(f)] show pseudocolor PA images superimposed on grayscale US echo images around the DIP (PIP) joint of the index finger. The PA and US echo images were reconstructed using the same methods as those of the phantom experiments using MATLAB. In the superimposed images, the contrast and brightness were manually enhanced as shown in Fig. 16(b).

In Figs. 16(a) and 17(a), the signals from the blood vessels (shown by red-dotted arrows) and bone (shown by yellowdotted arrows) were clearly visualized. Figure 16(a) shows that the system can visualize blood vessels that are $\sim 0.26 \mathrm{~mm}$ in diameter and at $\sim 4-\mathrm{mm}$ depth from the skin surface. Figure 16(d) is a superimposed image with a relatively strong PA signal from the skin, as shown by the cyan-solid arrow in Fig. 16(a), that was eliminated to highlight the signal from the blood vessels. However, in Fig. 17(d), the signal was not eliminated because of the weak signal in Fig. 17(a). The MIP images demonstrate that nearly the entire finger vasculature around the joint can be precisely mapped and its location can be clarified as the DIP and PIP joints are depicted by the white-solid arrows in Figs. 16(e), 16(f), 17(e), and 17(f). Figures 16 and 17 show that the system can visualize finger vasculature, bone structure, and the location of each joint. Therefore, it has been demonstrated that this study's ring-array system has the potential to image abnormal vascularization around a finger joint and it can be applied to inflammatory arthritis diagnosis.

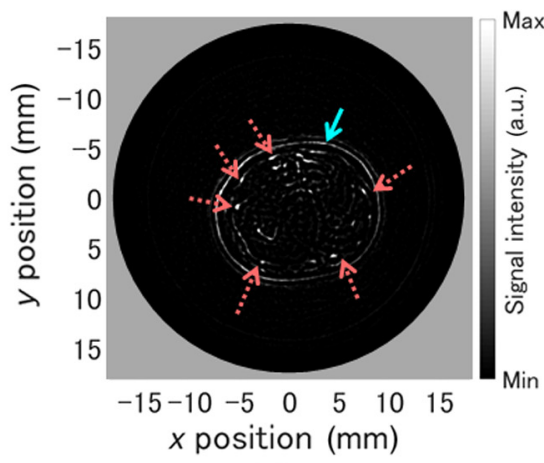

(a)

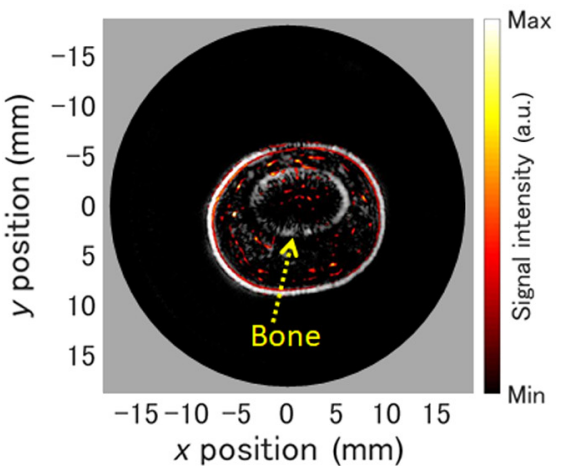

(d)

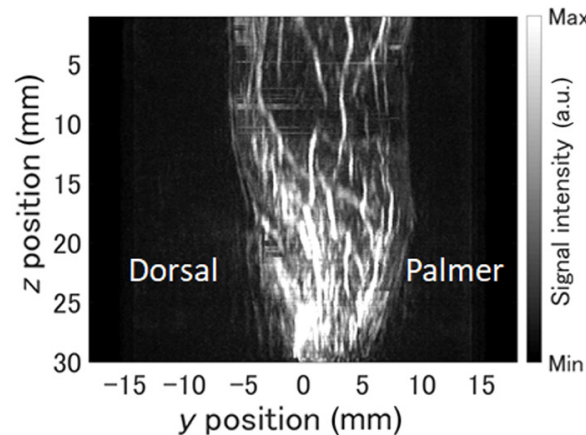

(b)

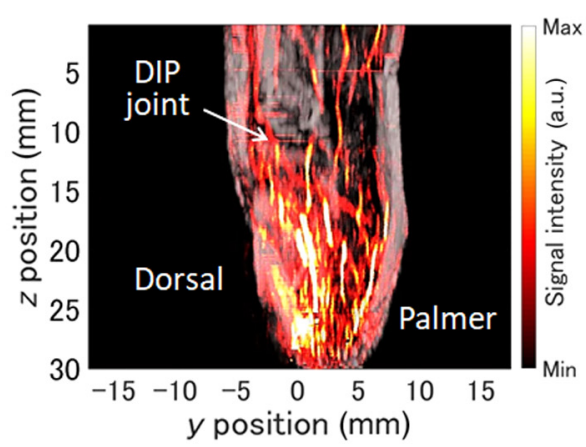

(e)

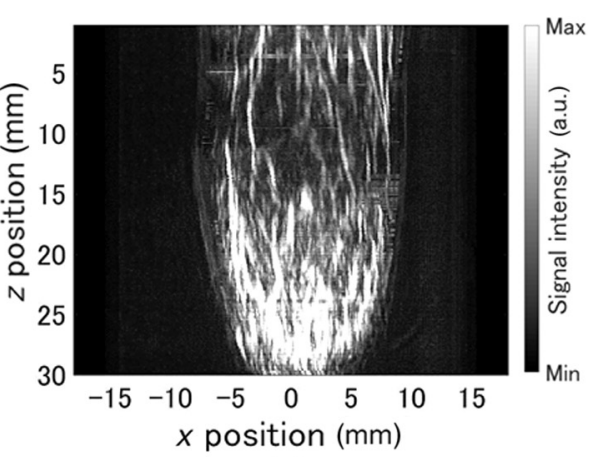

(c)

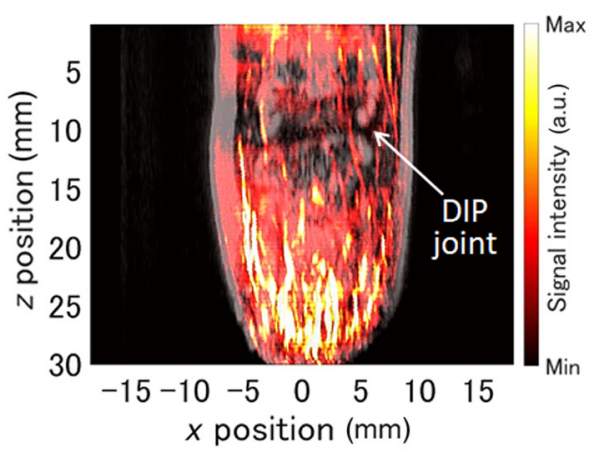

(f)

Fig. 16 Cross-sectional and MIP images around the DIP joint of a healthy index finger. The first row shows PA images and the second pseudocolor PA images superimposed on grayscale US images. (a), (d) Cross-sectional PA images. The red-dotted arrows show blood vessels, the cyan-solid arrow the skin surface, and the yellow arrows bone. (b), (e) MIP images of the lateral side of the joint. (c), (f) MIP images of the palmer side of the joint. In (e) and (f), the DIP joint is shown by the whitesolid arrows. Each MIP image was calculated from the skin surface to the center of the finger. 


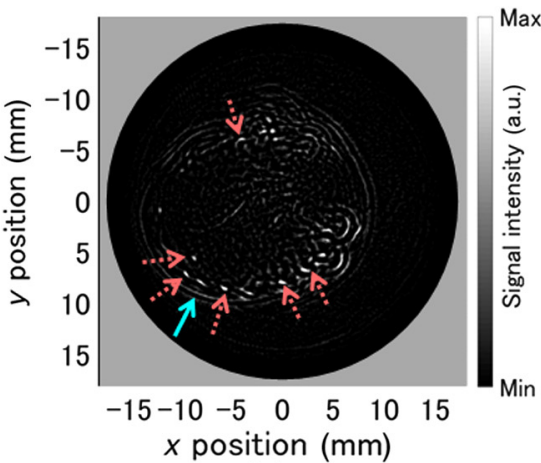

(a)

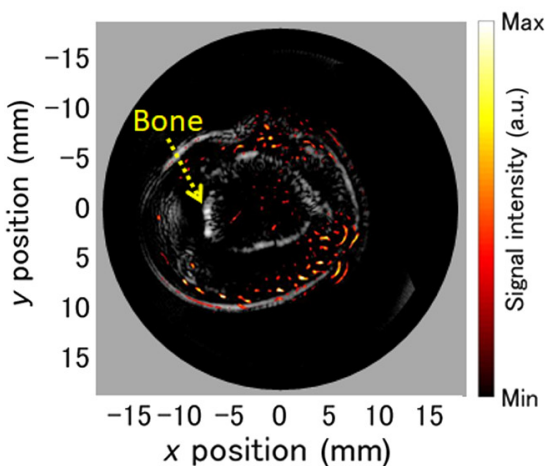

(d)

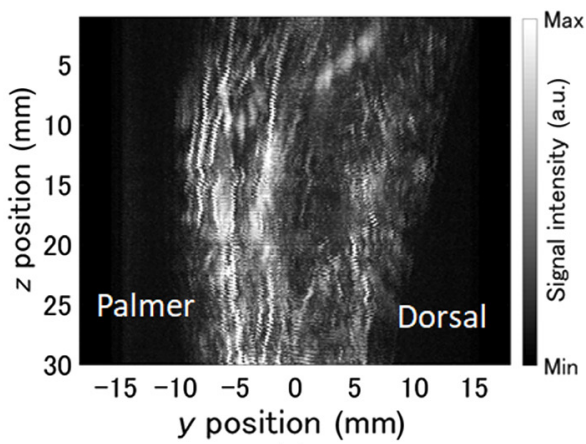

(b)

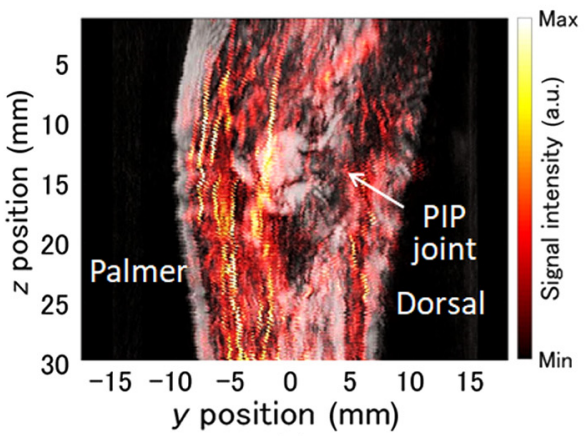

(e)

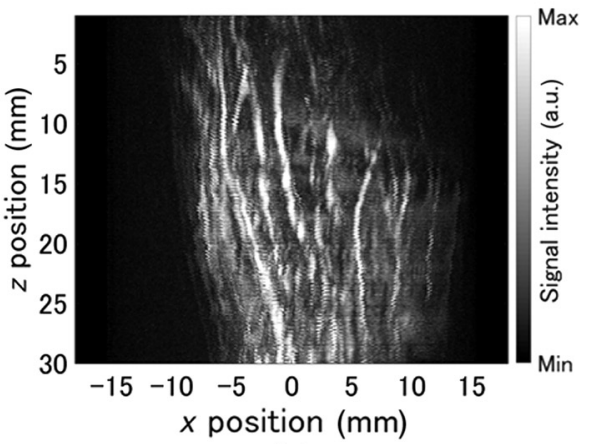

(c)

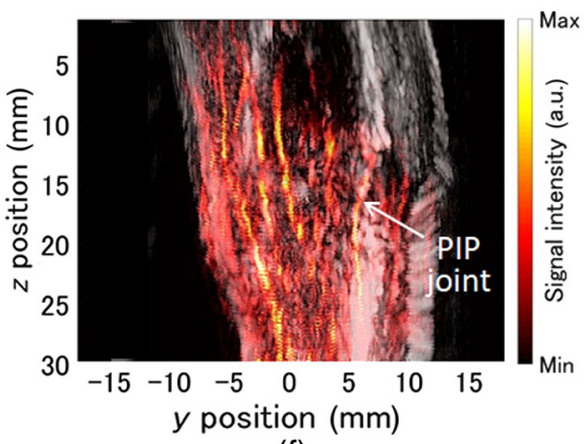

(f)

Fig. 17 Cross-sectional images and MIP images around the PIP joint of a healthy index finger. The first row shows PA images and the second pseudocolor PA images superimposed on grayscale US images. (a), (d) Cross-sectional PA images. The red-dotted arrows show blood vessels, the cyan-solid arrow the skin surface, and the yellow arrows bone. (b), (e) MIP images of the lateral side of the joint. (c), (f) MIP images of the dorsal side of the joint. In (e) and (f), the PIP is shown by the white-solid arrows.

\section{Discussions and Conclusions}

We were able to design a PA system using a ring-array sensor to image human finger vasculature. It was confirmed that the results of the phantom experiments were nearly the same as those of the simulation. Figures 11 and 12 show the applicability of a PA imaging system using the designed ring-array sensor. Figure 12 shows that the bone hardly affects the PA image as Fig. 4 also shows in this system. Figure 12 shows that this system can visualize smaller objects $\sim 0.1 \mathrm{~mm}$ in diameter using a ring-shaped optical illuminator. Figure 14(b) shows that an accurate 3-D image may be achieved while maintaining a high resolution regardless of the absorber's depth. In Figs. 16 and 17, we succeeded in mapping a finger's vascular structure that was $\sim 0.26 \mathrm{~mm}$ in diameter and at $\sim 4-\mathrm{mm}$ depth from the skin surface, as well as the DIP and PIP joints. However, part of the PA signal from the blood vessels was relatively weak and some artifacts around the skin were seen [particularly in Fig. 17(a)]. This was associated with the finger being slightly out of alignment with the axial direction during the measurement and the light illumination to the blood vessels being partly inadequate. This could be solved by refining the system such that the finger is tightly fixed in the center of the ring-array sensor and perpendicular to the cross section and by focusing the light illumination in the axial direction. In this study, the reconstruction algorithm used in all calculations assumes a constant sound of speed, which can cause some artifacts in the image. For more accurate images, utilizing algorithms that can reduce the artifacts induced by acoustic heterogeneity in the object, such as the half-time reconstruction algorithm, ${ }^{28,37}$ and optimizing one of the characteristics of a ring array, namely that it can measure sound distribution, can be effective.

Our system can simultaneously capture full-view PA and US signals from a human finger using the ring-array sensor, which can realize a more accurate image reconstruction with fewer artifacts than an arc-shaped transducer. ${ }^{20-24,26}$ In addition, this system is specialized in imaging finger vasculature using the ring-array sensor unlike other ring array studies dedicated to animal imaging ${ }^{28-32}$ and dual PAT and US studies using a linear array. ${ }^{17-19}$ Therefore, a more precise diagnosis of inflammatory arthritis is expected.

In conclusion, we sought to develop a PA system using a ring-shaped US transducer to image the finger vasculature. The ring-array sensor was designed based on simulations, and phantom experiments and finger imaging were conducted by experimentally producing the imaging system. Through 2-D and 3-D measurements of the phantoms, we determined that the system can map multiple absorbers, including those that are small (i.e., $\sim 0.2 \mathrm{~mm}$ in diameter), and attained an FWHM of 1.5 to $2 \mathrm{~mm}$ in the slice direction. It was confirmed via imaging of a healthy index finger that this system has the potential to help visualize an important marker of inflammatory arthritis (abnormal vascularization) because of its ability to image healthy human finger vasculature and joints by acquiring a coregistered PA and US image that is not affected by motion.

In this study, we presumed that the sound velocity was constant in the calculation. However, this system can more accurately visualize objects using algorithms considering the acoustic heterogeneity and measuring the sound distribution. 
In the future, through experiment and analysis using small animals and RA patients, we will develop a clinical PA imaging system that detects another important marker, hypoxia, as well as angiogenesis.

\section{Disclosures}

The authors declare that they have no conflicts of interest. This work is based on our prior proceedings: M. Nishiyama et al., "Development of photoacoustic imaging system of finger vasculature using ring-shaped ultrasound transducer," Proc. SPIE, Vol. 10494, Photons Plus Ultrasound: Imaging and Sensing 2018, Paper 1049448, May 2018 (doi: 10.1117/12.2288655).

\section{Acknowledgments}

This work was partly supported by the Impulsing Paradigm Change through the Disrupt Technologies (ImPACT) Program of the Cabinet Office of Japan and the Training Program of Leaders for Integrated Medical system (LIMS) at Kyoto University.

\section{References}

1. B. Heidari, "Rheumatoid arthritis: early diagnosis and treatment outcomes," Caspian J. Intern. Med. 2(1), 161-170 (2011).

2. J. E. Machado-Alba, A. F. Ruiz, and D. A. M. Morales, "The epidemiology of rheumatoid arthritis in a cohort of Colombian patients," Rev. Colomb. Reumatol. 22(3), 148-152 (2015).

3. J. R. Rajian et al., "Characterization and treatment monitoring of inflammatory arthritis by photoacoustic imaging: a study on adjuvantinduced arthritis rat model," Biomed. Opt. Express 4(6), 900-908 (2013).

4. A. K. Scheel et al., "Prospective 7 year follow up imaging study comparing radiography, ultrasonography, and magnetic resonance imaging in rheumatoid arthritis finger joints," Ann. Rheum. Dis. 65(5), 595-600 (2006).

5. M. Backhaus et al., "Arthritis of the finger joints: a comprehensive approach comparing conventional radiography, scintigraphy, ultrasound, and contrast-enhanced magnetic resonance imaging," Arthritis Rheum. 42(6), 1232-1245 (1999).

6. A. Marrelli et al., "Angiogenesis in rheumatoid arthritis: a disease specific process or a common response to chronic inflammation?" Autoimmun. Rev. 10(10), 595-598 (2011).

7. Z. Szekanecz and A. E. Koch, "Mechanisms of disease: angiogenesis in inflammatory diseases," Nat. Clin. Pract. Rheumatol. 3(11), 635-643 (2007).

8. M. A. Quinn et al., "Prognostic factors in a large cohort of patients with early undifferentiated inflammatory arthritis after application of a structured management protocol," Arthritis Rheum. 48(11), 30393045 (2003).

9. A. N. Colebatch et al., "EULAR recommendations for the use of imaging of the joints in the clinical management of rheumatoid arthritis," Ann. Rheum. Dis. 72(6), 804-814 (2013).

10. M. Xu and L. V. Wang, "Photoacoustic imaging in biomedicine," Rev. Sci. Instrum. 77(4), 041101 (2006).

11. L. Wang and $\mathrm{S}$. Hu, "Photoacoustic tomography: in vivo imaging from organelles to organs," Science 335(6075), 1458-1462 (2012).

12. Y. Zhou, J. Yao, and L. V. Wang, "Tutorial on photoacoustic tomography," J. Biomed. Opt. 21(6), 061007 (2016).

13. P. Beard, "Biomedical photoacoustic imaging," Interface Focus 1(4), 602-631 (2011).

14. J. Xia, J. Yao, and L. V. Wang, "Photoacoustic tomography: principles and advances," Electromagn. Waves 147, 1-22 (2014).

15. D. Golovko et al., "Optical imaging of rheumatoid arthritis," Int. J. Clin. Rheumatol. 6(1), 67-75 (2011).

16. D. Chamberland, Y. Jiang, and X. Wang, "Optical imaging: new tools for arthritis," Integr. Biol. 2(10), 496-509 (2010).

17. G. Xu et al., "Photoacoustic and ultrasound dual-modality imaging of human peripheral joints," J. Biomed. Opt. 18(1), 010502 (2012).
18. J. Jo et al., "A functional study of human inflammatory arthritis using photoacoustic imaging," Sci. Rep. 7(1), 1-9 (2017).

19. P. J. van den Berg et al., "Feasibility of photoacoustic/ultrasound imaging of synovitis in finger joints using a point-of-care system," Photoacoustics 8, 8-14 (2017).

20. P. van Es et al., "Initial results of finger imaging using photoacoustic computed tomography," J. Biomed. Opt. 19(6), 060501 (2014).

21. S. K. Biswas et al., "A new approach to depict bone surfaces in finger imaging using photoacoustic tomography," Proc. SPIE 9323, 932322 (2015).

22. P. van Es et al., "Photoacoustic tomography of the human finger: towards the assessment of inflammatory joint diseases," Proc. SPIE 9323, 93234Q (2015).

23. S. K. Biswas et al., "A method for delineation of bone surfaces in photoacoustic computed tomography of the finger," Ultrason. Imaging 38(1), 63-76 (2015).

24. E. Merčep et al., "Hybrid optoacoustic tomography and pulseecho ultrasonography using concave arrays," IEEE Trans. Ultrason. Ferroelectr. Freq. Control 62(9), 1651-1661 (2015).

25. Z. Deng, "Noninvasively measuring oxygen saturation of human fingerjoint vessels by multi-transducer functional photoacoustic tomography," J. Biomed. Opt. 21(6), 061009 (2016).

26. Y. Sun, E. S. Sobel, and H. Jiang, "First assessment of threedimensional quantitative photoacoustic tomography for in vivo detection of osteoarthritis in the finger joints," Med. Phys. 38(7), 4009-4017 (2011).

27. M. Oeri et al., "Hybrid photoacoustic/ultrasound tomograph for real-time finger imaging," Ultrasound Med. Biol. 43(10), 2200-2212 (2017).

28. J. Xia et al., "Whole-body ring-shaped confocal photoacoustic computed tomography of small animals in vivo," J. Biomed. Opt. 17(5), 050506 (2012).

29. L. Li et al., "Single-impulse panoramic photoacoustic computed tomography of small-animal whole-body dynamics at high spatiotemporal resolution," Nat. Biomed. Eng. 1(5), 0071 (2017).

30. J. Xia et al., "Anatomical and metabolic small-animal whole-body imaging using ring-shaped confocal photoacoustic computed tomography," Proc. SPIE 8581, 85810K (2013).

31. H.-P. Brecht et al., "Whole-body three-dimensional optoacoustic tomography system for small animals," J. Biomed. Opt. 14(6), 064007 (2009).

32. C. Yeh et al., "Dry coupling for whole-body small-animal photoacoustic computed tomography," J. Biomed. Opt. 22(4), 041017 (2017).

33. J. Xia et al., "Enhancement of photoacoustic tomography by ultrasonic computed tomography based on optical excitation of elements of a full-ring transducer array," Opt. Lett. 38(16), 3140-3143 (2013).

34. B. E. Treeby and B. T. Cox, "k-Wave: MATLAB toolbox for the simulation and reconstruction of photoacoustic wave fields," J. Biomed. Opt. 15(2), 021314 (2010).

35. M. Xu and L. V. Wang, "Universal back-projection algorithm for photoacoustic computed tomography," Phys. Rev. E 71(1), 016706 (2005).

36. X. Qu et al., "Synthetic aperture ultrasound imaging with a ring transducer array: preliminary ex vivo results," J. Med. Ultrason. 43(4), 461-471 (2016).

37. M. A. Anastasio et al., "Half-time image reconstruction in thermoacoustic tomography," IEEE Trans. Med. Imaging 24(2), 199-210 (2005).

Misaki Nishiyama received her BS degree in laboratory science from Doshisha University, Japan, in 2016 and her MS degree from Kyoto University, Japan, in 2018. Currently, she is working toward her PhD at Kyoto University. Her research interests include medical imaging related to photoacoustics, ultrasonics, and optics.

Takeshi Namita received his PhD from the Hokkaido University, Japan, in 2009. From 2009 to 2013 he was a postdoctoral fellow in the Graduate School of Information Science and Technology at the Hokkaido University, Japan. Currently, he is a program-specific assistant professor in the Graduate School of Medicine at Kyoto University, Japan. He has been engaged in studies of biomedical engineering, including those of diffused optical tomography and photoacoustic imaging in biological media. 
Kengo Kondo received his $\mathrm{BE}, \mathrm{ME}$, and $\mathrm{PhD}$ degrees in engineering from University of Tsukuba in 2005, 2007 and 2010, respectively. Currently, he is a program-specific assistant professor in the Graduate School of Medicine, Kyoto University. His research interests include ultrasound and photoacoustic imaging, signal processing, and tissue characterization.

Makoto Yamakawa received his $\mathrm{MS}$ and $\mathrm{PhD}$ degrees from the University of Tsukuba, Japan, in 1999 and 2002, respectively. Currently, he is an associate professor in the Graduate School of Medicine, Kyoto University. His current research focuses on the photoacoustic imaging, tissue elasticity imaging, and artificial intelligence for ultrasound imaging.

Tsuyoshi Shiina received his BS, MS, and PhD degrees in electronic engineering from the University of Tokyo in 1982, 1984, and 1987, and received DMSC degree from University of Tsukuba in 2006. From 1995 to 1996, he was with the Institute of Cancer Research and the Royal Marsden NHS Trust in UK as visiting professor. Recently, he involved the development of photoacoustic imaging equipment as the ImPACT Program supported by a Japanese government grant. 